Revue des patrimoines

43 | 2021

Des écoles d'art académiques aux écoles d'art : des collections et des lieux, un patrimoine à valoriser

\title{
BMBF-Projekt „Körper und Malerei“: Erschließung, Erforschung und Nutzung der Anatomischen Lehrsammlung und der Gemäldesammlung der Hochschule für Bildende Künste Dresden
}

To exhibit the anatomical collection of the Dresden University of Fine Arts: the German federal project "Körper und Malerei"

Sandra Mühlenberend, Jakob Fuchs et Ivo Mohrmann

\section{(2) OpenEdition}

Édition électronique

URL : http://journals.openedition.org/insitu/30131

DOI : 10.4000/insitu.30131

ISSN : 1630-7305

Éditeur

Ministère de la Culture

Référence électronique

Sandra Mühlenberend, Jakob Fuchs et Ivo Mohrmann, «BMBF-Projekt „Körper und Malerei“:

Erschließung, Erforschung und Nutzung der Anatomischen Lehrsammlung und der

Gemäldesammlung der Hochschule für Bildende Künste Dresden », In Situ [En ligne], 43 | 2021, mis en ligne le 02 février 2021, consulté le 03 février 2021. URL : http://journals.openedition.org/insitu/30131 ; DOI : https://doi.org/10.4000/insitu.30131

Ce document a été généré automatiquement le 3 février 2021

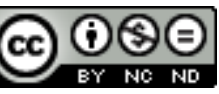

In Situ Revues des patrimoines est mis à disposition selon les termes de la licence Creative Commons Attribution - Pas d'Utilisation Commerciale - Pas de Modification 4.0 International. 


\section{BMBF-Projekt „Körper und Malerei“: Erschließung, Erforschung und Nutzung der Anatomischen Lehrsammlung und der Gemäldesammlung der Hochschule für Bildende Künste Dresden}

To exhibit the anatomical collection of the Dresden University of Fine Arts: the German federal project "Körper und Malerei"

Sandra Mühlenberend, Jakob Fuchs et Ivo Mohrmann

\section{Hochschule für Bildende Künste Dresden}

1 Die Hochschule für Bildende Künste Dresden (HfBK) gehört $\mathrm{zu}$ den ältesten Kunstakademien Europas. Bereits seit dem Jahr 1680 existierte die „Zeichen- und Malerschule“ als Vorläufereinrichtung. 1764 erfolgte die Gründung der Hochschule unter dem Namen „Haupt-Kunst-Akademie“. Bereits kurze Zeit nach ihrer Gründung konnte die Kunstakademie national und international renommierte Lehrende für die Ausbildung der Studierenden gewinnen. $\mathrm{Zu}$ den bekanntesten zählen u.a. Charles François Hutin, Bernardo Bellotto, Giuseppe Camerata, Giovanni Battista Casanova und Lorenzo Zucchi sowie Anton Graff und Adrian Zingg.

2 Im 19. Jahrhundert zog die Akademie zahlreiche bekannte Künstler, wie Caspar David Friedrich, Philipp Otto Runge und Georg Friedrich Kersting nach Dresden. Besonders hervorzuheben ist das lange und bedeutungsvolle Wirken Ludwig Richters, der zunächst an der Akademie studierte und ab 1836 Malerei lehrte. Der Architekt Gottfried 
Semper, der in der ersten Hälfte des 19. Jahrhunderts maßgeblich das Stadtbild Dresdens mitprägte, wurde 1834 Professor der Architekturabteilung.

3 In der zweiten Hälfte des 19. Jahrhunderts folgte eine Phase der Stagnation. Um sich aus der Erstarrung der akademisierten Ausbildung zu lösen, berief die Kunstakademie im Jahre 1895 den Impressionisten Gotthardt Kuehl zum Professor für Malerei; es folgten bedeutende Professoren, wie Otto Gussmann, Carl Bantzer, Eugen Bracht, Robert Sterl, Ferdinand Dorsch und Studenten, wie Max Pechstein, Kurt Schwitters, George Grosz, Conrad Felixmüller und Otto Dix. Innerhalb weniger Jahre konnte sich die Kunstakademie vollständig erneuern und ihre Bedeutung in den 1920er Jahren zusätzlich durch die Berufung von Oskar Kokoschka und Otto Dix steigern. ${ }^{1}$

4 Nach dem Zweiten Weltkrieg nahm die Akademie 1947 ihre Lehrtätigkeit wieder auf und wurde 1950 zusammen mit der Kunstgewerbeschule zur „Hochschule für Bildende Künste" vereinigt. Danach galt es, die Kunsthochschule als künstlerische Ausbildungsstätte des sozialistischen Realismus zu etablieren. Bis 1990 wurden mehrere Reformen angestrengt, immer in Verbindung mit gesellschaftlichen Vorgaben und Auflagen. Im Zentrum stand weiter die figürliche Kunst; erst gegen Ende der 1980 Jahre konnte auch abstrakt gearbeitet werden. ${ }^{2}$

\section{BMBF-Forschungsprojekt „Körper und Malerei“}

5 Unter der Überschrift „Vernetzen- Erschließen - Forschen“ hat das deutsche Bundesministerium für Bildung und Forschung (BMBF) 2016 eine Allianz für universitäre Sammlungen ins Leben gerufen. Dank einer Förderung aus diesem Programm konnten zwei, in ihrer Art einzigartige Sammlungsbestände der Hochschule für Bildende Künste Dresden (HfBK Dresden) aus ihrem Schattendasein gehoben werden: Die mit ca.700 Modellen, Präparaten und Lehrtafeln ausgestattete Anatomische Sammlung, kontinuierlich zusammengetragen seit 1800, und die ca. 1.400 umfassende Gemäldesammlung von künstlerischen Diplombeleg- und Studienarbeiten, entstanden in der Zeit von 1950 bis 1990.

6 Das Projekt „Körper und Malerei“ beinhaltete u.a. die Bestands- und Zustandserfassung, konservatorische sowie restauratorische Maßnahmen und fokussierte die Erforschung, Sicherung und Vermittlung dieser beiden in ihrer historischen Bedeutung und aktuellen Relevanz bisher wenig beachteten Sammlungen. Zudem verfolgte das Projekt das Anliegen, die Sammlungen über die dreijährige Projektlaufzeit hinaus mittels einer zu entwickelnden, zeitgemäßen Infrastruktur für die Wissenschaft und die Lehre zugänglich und nutzbar zu machen. Während für die anatomische Sammlung zwar ein erstes Überblickswerk zu einem Großteil der wertvollen Objekte und ihrer historischen Verwendung existiert ${ }^{3}$, lagen die Potentiale der Gemäldesammlung für Forschung und Lehre bisher nahezu brach. Der Stellenwert der künstlerischen Diplomübereignungen an die Hochschule und der Studienarbeiten wurde bisher weder in der Kunstgeschichte zur Malerei in der DDR noch in der Forschung zur Institutionengeschichte wahrgenommen. Auch die Arbeit an und mit der Gemäldesammlung setzte sich zum Ziel, diese in Forschung und Lehre zu vitalisieren.

7 Hochaktuelle Fragen zur Körpergeschichte und den ethischen Umgang mit historischen menschlichen Präparaten, zu Ausbildungsstrategien an Kunsthochschulen, zur Materialität, Geschichte und Bedeutung von Objekten in wissenschaftlichen und künstlerischen Sammlungskontexten und ihre komplexen Verbindungen standen 
hierbei stets im Fokus. Begleitet und betreut wurde das dreijährige Projekt durch den Kooperationspartner Staatliche Kunstsammlungen Dresden (SKD). Die praktische und theoretische Erschließung der beiden Sammlungen erfolgte im interdisziplinären Dialog von Kunst- und Wissenschaftsgeschichte, Konservierung/ Restaurierung, naturwissenschaftlicher Analytik sowie Sammlungsmanagement.

\section{Anatomische Sammlung}

Die Anatomiesammlung der Hochschule für Bildende Künste in Dresden gehört zu den wertvollsten und umfangreichsten ihrer Art. An ihr können alle wichtigen Schwerpunkte der Künstleranatomie in den letzten 200 Jahren demonstriert werden sei es zur Human- wie auch zur Tieranatomie [fig. 1, fig. 2]. Ihre von ca. 700 Objekten mit über 1.000 Einzelteilen ausgehenden Impulse treffen auf aktuelle künstlerische wie wissenschaftliche Forschung. Die Erhaltung der Sammlung begründet sich auf mehreren Umständen: der kontinuierlichen Lehre der Künstleranatomie seit 1800 bis heute, ihrem hohen Stellenwert an der Dresdner Kunstakademie bis in die 1980er Jahre hinein sowie einem professionellen und engagierten Personal aus Medizin und Kunst.

Figur 1

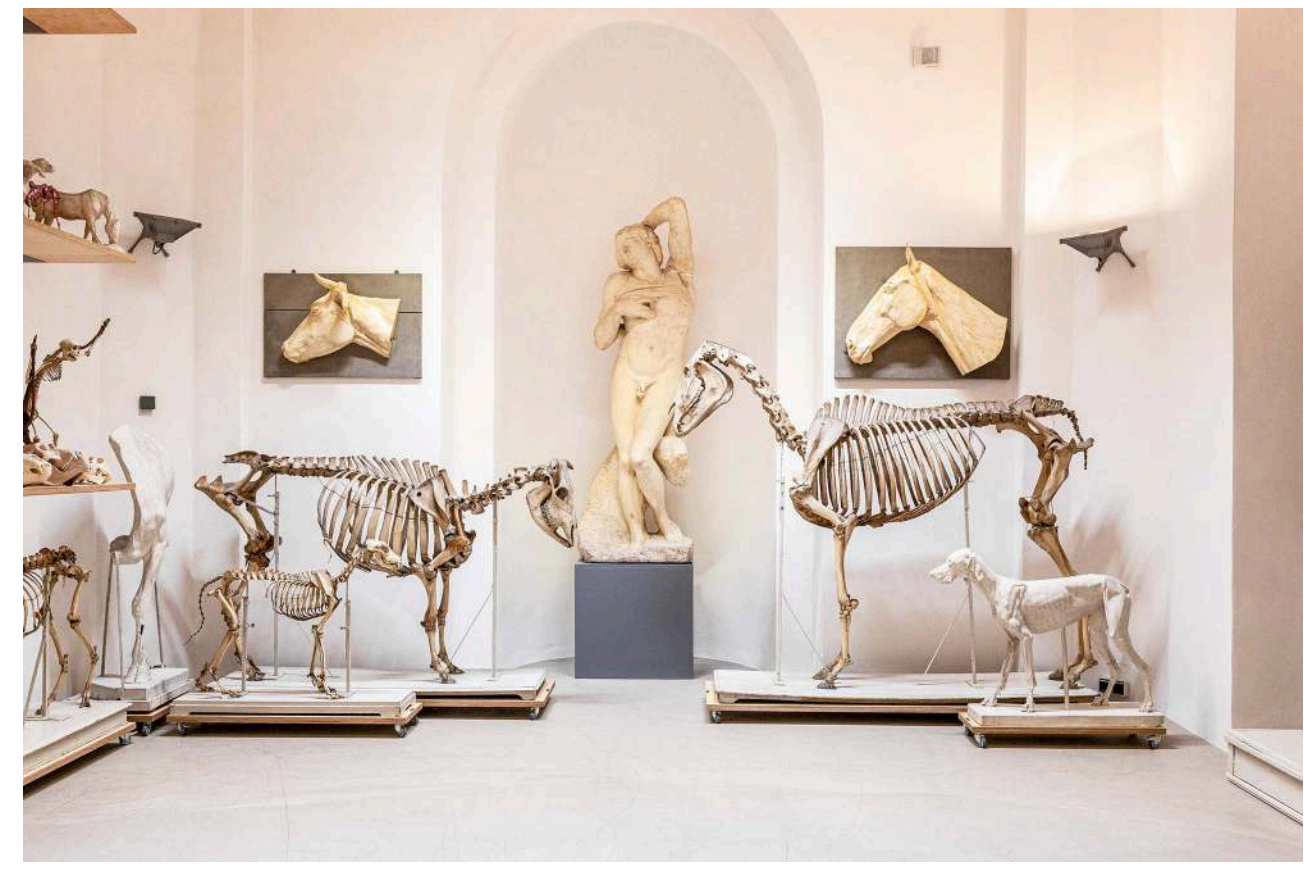

Modelle und Präparate zur Tieranatomie sowie ein Gipsabguss des, sterbenden, Sklaven“ von Michelangelo Buonarotti.

(C) Robert Vanis.

In der europäischen Geschichte der Künstleranatomie waren von Beginn an die Disziplinen Kunst und Medizin eng verbunden. Entweder arbeiteten zwei jeweilige Vertreter zusammen oder die Interessen und Begabungen jedes einzelnen deckten das Fach des anderen mit ab. Auch an der Kunstakademie Dresden lässt sich für das kunstanatomische Fach diese besondere Verbindung ablesen. Zuerst wurde es von Künstlern wie Giovanni Battista Casanova (1730-1795) unterrichtet. Dieser beschäftigte sich aus eigenem künstlerischem Interesse mit dem inneren Aufbau des Körpers und 
formulierte auf dieser Grundlage ein Lehrkonzept. Später folgte der seinerzeit berühmte Anatom und Mediziner Burkhard Wilhelm Seiler (1779-1843), der sich den Künsten nicht nur zuwandte, sondern auch ein Lehrbuch konzipierte, welches sein Wissen mit dem damaligen Kunstgeschmack verband. Beide - Casanova und Seiler konsultierten jeweils Vertreter der gegenüberliegenden Disziplin, ließen sich beraten und konnten so eine ganz eigene Vermittlungsstrategie entwickeln. Die personelle Besetzung des Faches zeigt von dessen Gründung an, dass jene Professoren besonders nachhaltig arbeiteten, die beide Disziplinen gleichermaßen studierten und zeitgemäße Verknüpfungspunkte suchten und visualisierten. Als Protagonisten führten Burkhard Wilhelm Seiler, Hermann Dittrich (1868-1946), Gottfried Bammes (1920-2007) und Manfred Zoller (*1947) die Künstleranatomie an der Dresdner Kunstakademie, später der Hochschule, zum nachhaltigen Erfolg. Sie sind einerseits verantwortlich für das Wachsen der Sammlung durch Ankäufe und durch Eigenkonzeptionen anatomischer Lehrobjekte, andererseits entwickelten sie neue Lehrkonzepte, die weit über die Akademie hinausstrahlten und große Anerkennung fanden. ${ }^{4}$

Figur 2

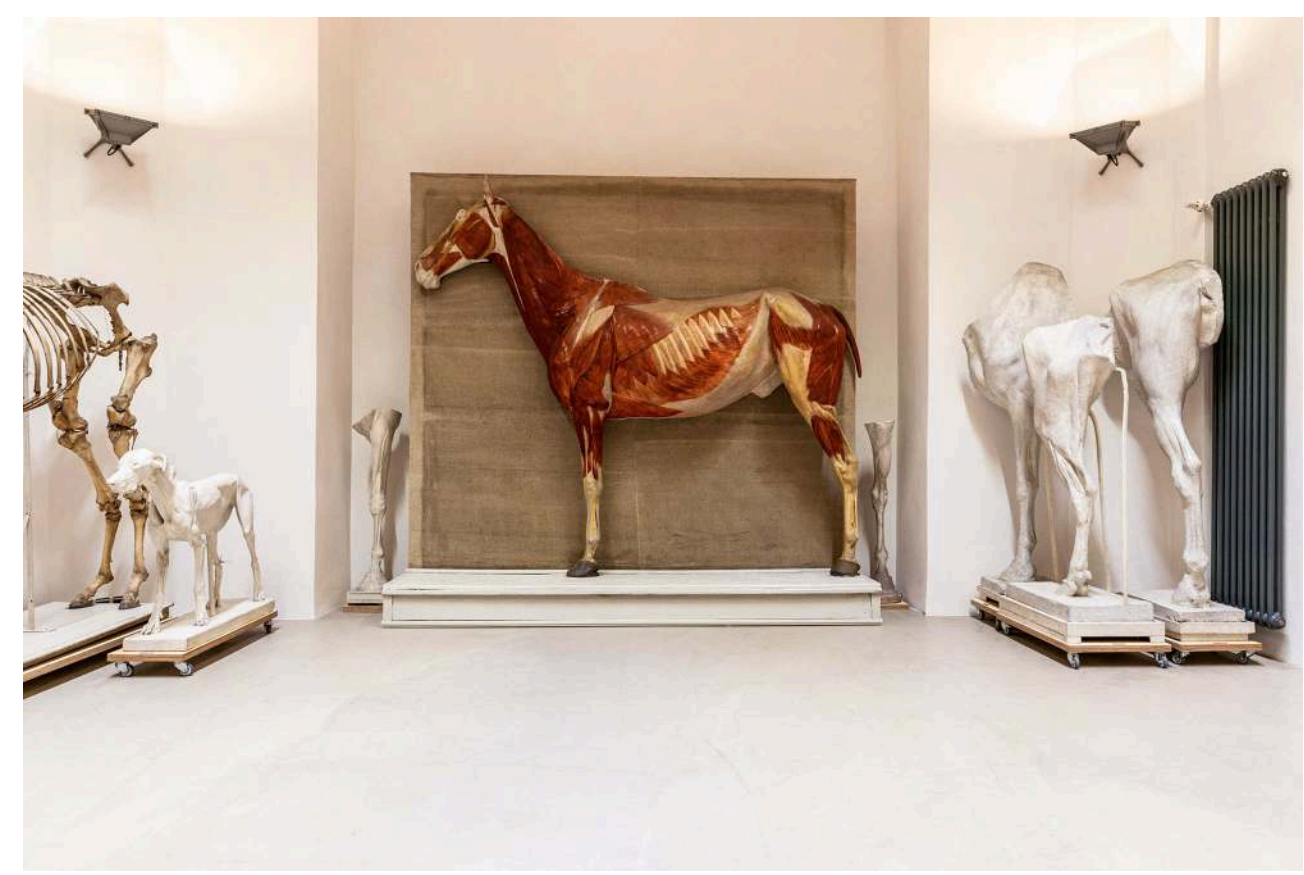

Lebensgroßes farbig gefasstes Pferderelief in Gips, wahrscheinlich hergestellt in Zusammenarbeit mit der Tierärztlichen Hochschule Dresden zwischen 1900 und 1920.

(c) Robert Vanis.

Nur ein Abschnitt der Geschichte wirkt ungeordnet und war personell schwach besetzt: die zweite Hälfte des 19.Jahrhunderts, also jene Zeit, in der Reformen mehr als überfällig waren und die gesamte Kunstakademie in einer gewissen Erstarrung verharrte. Während der NS-Zeit wich die Künstleranatomie von der befruchtenden Verbindung beider Disziplinen ab, eher stand die Bestätigung überhöhter Körperhüllen zu Propagandazwecken im Mittelpunkt. In die Zeit von 1933 bis 1945 fällt auch das dunkelste Kapitel der Anatomischen Sammlung, das nicht von ungefähr in Verbindung mit den Schrecken des Nationalsozialismus steht. Von 1933 bis in das Jahr 1942 unterrichtete der Künstler Fritz Otto Sauerstein (1893-1968) das Fach. Er gestaltete den 
Unterricht gemäß den Zielen der nationalsozialistischen Ideologie und führte einen Lehrgang für Rassenkunde und Rassenpflege ein. Wie Dittrich sezierte er Leichen, die bis dahin vom Wohlfahrtspolizeiamt des Rates zu Dresden überführt wurden. Hierbei handelte es sich um Verstorbene, für die sich verwandtschaftlich niemand verantwortlich fühlte. ${ }^{5}$

11 Sauerstein forderte jedoch auch Leichname von der Hinrichtungsstätte am Münchner Platz an. Nachweislich hat er 1936 von dort eine Leiche erhalten, vermutlich geschah dies auch 1941. Was mit den Leichnamen nach der Sektion passierte, kann nicht rekonstruiert werden. Untersuchungen der Vorgänge erfolgten 2014 und 2017 und deren Ergebnisse wurden in Forschungsberichten festgehalten. Sie führten zu einer Neubewertung der Präparate und zum Senatsbeschluss, alle losen und nicht zuzuordnenden menschlichen Überreste $\mathrm{zu}$ bestatten und nur jene Skelettaufstellungen der Sammlung zu zeigen, deren Herkunft außerhalb dieses Unrechtskontextes steht. ${ }^{6}$

$\mathrm{Zu}$ den im Sinne eines Unrechtskontextes unbelasteten Präparaten zählen beispielsweise die weltweit einzigartigen sieben Bänderskelette ${ }^{7} \mathrm{im}$ Gestus antiker Bildwerke, die in der ersten Hälfte des 19. Jahrhunderts hergestellt wurden. Sie stammen aus der ehemaligen Sammlung der Königlich Chirurgisch-Medicinischen Akademie und wurden nach Auflösung der Sammlung 1864 der Kunstakademie übereignet. Die sehr aufwendigen Präparationen und die besonderen Präsentationen gehen vermutlich auf Burkhard Wilhelm Seiler zurück. Die Skelettdarstellungen markieren den Höhepunkt der akademisch künstlerischen Auseinandersetzung mit dem antiken Schönheitsideal und den besonderen Proportionsverhältnissen antiker Skulpturen. Die Bänderskelette wurden für das Antikenstudium in Haltungen bevorzugter „Exempla“ gebracht - zum Beispiel des Borghesischen Fechters für den stark bewegten Körper, der Laokoongruppe (Laokoon-Skelett verschollen; erhalten sind die beiden Kinderskelette) für Emotion und Leidenschaft, der Venus Medici für Schönheit der weiblichen Figur und des Dornausziehers für Ruhe und Konzentration. Die präparatorisch erfolgreichen Umsetzungen dienen hier dem Nacheifern und Nachstellen der damals verehrten antiken Vorbilder als Vorwegnahme eines ästhetischen Ziels. ${ }^{8}$

13 Neben diesen bedeutenden Präparaten sowie Modellen zur Humananatomie, wie beispielsweise lebensgroße Muskelabformungen in Gips und seltene Kopfanatomien in Wachs, enthält die Sammlung zudem eine der aussagekräftigsten Kollektionen zur künstlerischen Tieranatomie. Einige dieser Objekte wurden von Lehrmittelfirmen [fig. 3] angekauft oder von Dresdner Institutionen übereignet. ${ }^{9}$ Außerdem fertigten auch Lehrende selbst originäre Modelle und Präparatereihen, bisweilen thematisch noch einmal erweitert in Gips und fotografisch sowie grafisch und publizistisch dokumentiert. 
Figur 3

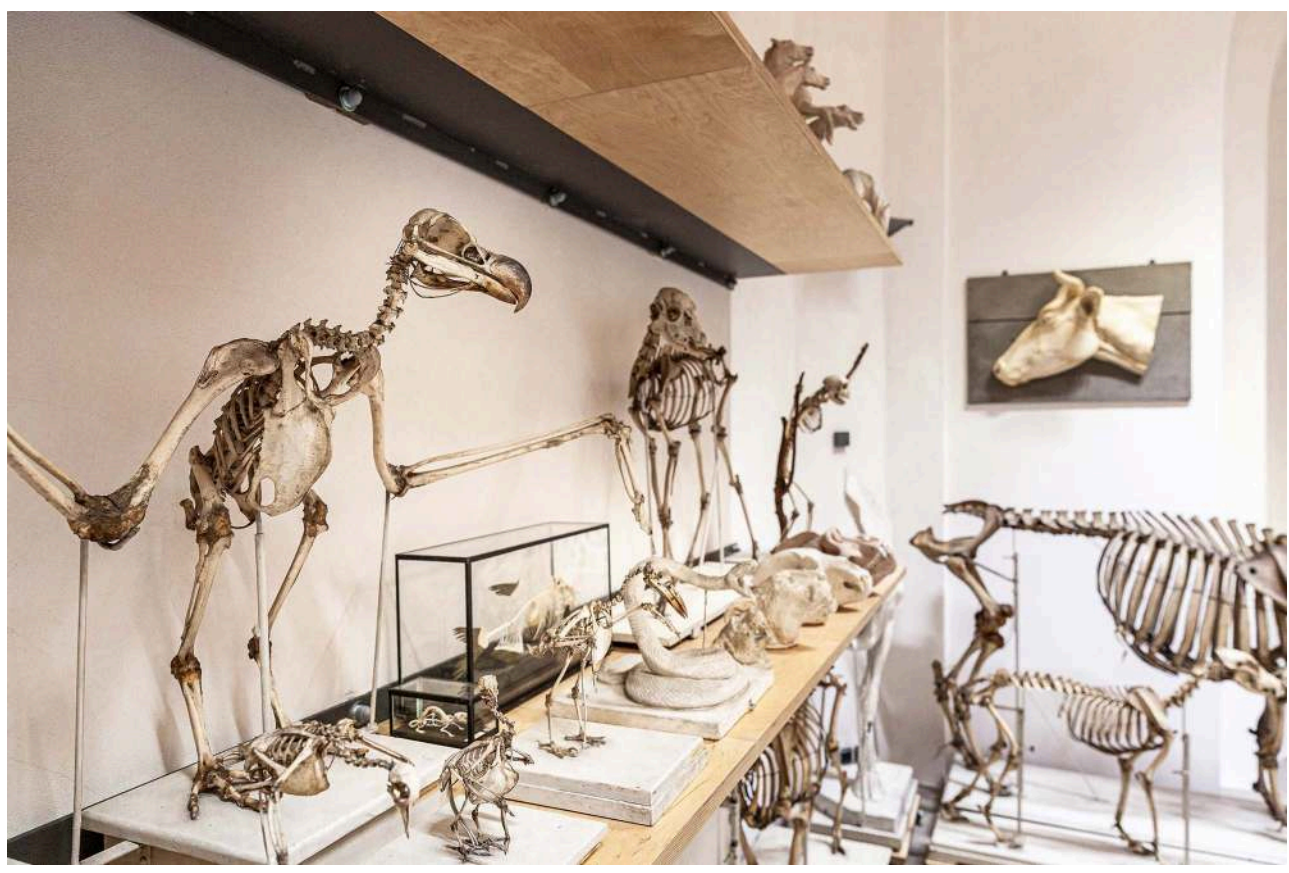

Modelle und Präparate zur Tieranatomie; im Vordergrund verschiedene Vogelskelette (c) Robert Vanis.

Ein farbig gefasster, lebensgroßer Muskeltorso aus Gips ist ein Originalabguss der berühmten Bronzeplastik von Carl Schütz (Lebensdaten unbekannt), die sich im Institut für Anatomie der Charité in Berlin befindet. Dort fand auch die Vormodellierung der Plastik statt, wobei Originalpräparate (Leichname) aus dem Institut zu Hilfe genommen wurden. An der Entstehung der Plastik war unter anderem der Anatom Hans Virchow (1852-1940) beteiligt, der die Anfertigung 1896 dokumentierte. Sie verlief nicht immer reibungslos und dauerte zwölf Jahre in Zusammenarbeit mit weiteren Künstlern und Anatomen. Der Gipsabguss war im Unterschied zu der Bronzeplastik direkt als Lehrmittel für Bildungseinrichtungen vorgesehen. Die linke Rumpfseite zeigt die Muskulatur direkt unter der Hautschicht, die rechte Seite tiefer liegende Strukturen - am Kopf und an den Oberschenkeln ist es umgekehrt. ${ }^{10}$

Die Kopie eines Naturabgusses von einem präparierten Tiger entstand um 1900 in München in der Werkstatt des Bildhauers Willy Zügel (1876-1950). Neben seinen künstlerischen Bildhauerarbeiten fertigte Zügel zahlreiche anatomische Naturabgüsse von Tieren in Gips, die er in Kopien verschiedenen Bildungseinrichtungen anbot. Der Muskeltiger ist dabei herausragend und das erste Modell seiner Art; vor allem Kunstakademien erwarben ihn für ihre anatomischen Sammlungen und als Modell für Tierdarstellungen.

Der Gipsabguss in Dresden ist nach derzeitigem Kenntnisstand der einzige Abguss von den verkauften Kopien (beispielsweise an die Kunstakademien Berlin und München), der noch erhalten geblieben ist; auch der Naturabguss selbst ist verschollen. Der Muskeltiger besticht nicht nur wegen seines Seltenheitswertes, sondern auch durch seine Ausführung - Schrittstellung, geöffnetes Maul, angespannte Muskeln-, die aus dem ursprünglich vorliegenden Kadaver ein lebendiges, aggressives Raubtier macht. ${ }^{11}$ 
17 In der Geschichte anatomischer Lehrobjekte spielt Wachs eine wichtige Rolle. Seine materielle Eigenschaft, die es ermöglicht, Organisches täuschend ähnlich nachzubilden, faszinierte Künstler wie Anatomen gleichermaßen. Es wurde bevorzugt bis ins 19. Jahrhundert hinein für den anatomischen Modellbau eingesetzt. Die Sammlung besitzt insgesamt 48 anatomische Wachsmodelle: 11 Wachsmodelle unbekannter Herkunft zu den oberen und unteren Extremitäten, 22 anatomische Wachsreliefs nach Josef Benedikt Kuriger (1754-1819) und 15 Kopfanatomien aus der Werkstatt von Carl Friedrich H. Heinemann (1802-1846). Die Wachsreliefs und Kopfanatomien wurden ebenfalls 1864, nach Auflösung der Sammlung der Königlich Chirurgisch-Medicinischen Akademie der Kunstakademie übereignet.

Die Modelle zu Arm und Bein sind klassische Studienobjekte in der Künstleranatomie. Ihre sehr genaue Ausführung regte an, sie für den Unterricht und ständigen Gebrauch zahlreich in Gips zu reproduzieren. Inwieweit die Wachsreliefs und Kopfanatomien dann auch zum Einsatz im kunstanatomischen Unterricht kamen, kann nur vermutet werden. Die Wachsreliefs, um 1800 entstanden, verdeutlichen zwar anatomische Zusammenhänge, jedoch eher in der Art eines Kunstkammerstücks. Ganz anders die Kopfanatomien, um 1830 für Mediziner gefertigt, die feinste anatomische Strukturen in ausgewählten Kopfbereichen lebens- oder überlebensgroß wiedergeben. ${ }^{12}$

Neben dem Bestand an zum Teil einzigartigen Präparaten und Modellen, begründet sich die nationale wie internationale Exklusivität der Anatomischen Sammlung auch dadurch, dass vergleichbare historische Sammlungen an anderen Kunstakademien verloren gegangen sind. Ausnahmen bilden lediglich die Sammlung der École nationale supérieure des Beaux-Arts de Paris und die der Russischen Kunstakademie St. Petersburg.

Die Wiederentdeckung der Dresdener Anatomiesammlung, die Ende der 1990er Jahre im Rahmen der Dissertation von Dr. Sandra Mühlenberend ${ }^{13}$ einsetzte und zu einer ersten Bestandsicherung führte, ging ähnlich wie in Paris und St. Petersburg mit dem Wunsch einher, sie mit ihren vielfältigen und sehr detailtreuen objekten in heutiger Zeit kaum noch vorzufindender Anschaulichkeit zu sichern und wieder für Lehre und Forschung nutzbar zu machen.

21 Bevor die Anatomiesammlung als Wissenschaftsinstrument / Lehrsammlung in der HfBK Dresden wieder genutzt und der Öffentlichkeit in Form einer Schausammlung vorgestellt werden konnte, musste sie aus dem Zustand der ungeordneten, zum Teil ungesicherten Aufbewahrung in eine sachgemäße Form überführt werden. Dies bedeutete, den Gesamtbestand $\mathrm{zu}$ sichten, diesen $\mathrm{zu}$ inventarisieren, die Objektzustände u.a. durch Materialanalytik zu erfassen und auf dieser Grundlage Maßnahmen der Konservierung und Restaurierung einzuleiten.

Als erste Maßnahme musste der gesamte Sammlungsbestand aus den beiden miteinanderverbundenen Haupträumen ausgelagert werden. Da massive Staub- und Schmutzablagerungen vor allem auf das unverputzte Ziegelmauerwerk zurückzuführen waren, wurde die gesamte Oberfläche des Innenraums verputzt. Es folgten die veränderte Anbringung der Wandregale und die Installation einer Arbeitsplatz- sowie Ausstellungsbeleuchtung.

Um alle 468 Präparate und Modelle der Sammlung konservatorisch zu erfassen und den Zustand zu dokumentieren, musste zunächst ein Protokollformular entwickelt werden. Dieses sollte für den gesamten Sammlungsbestand nutzbar sein und neben der 
Erfassung von Kerndaten und Schäden auch eine kurze Schadensanalyse (Grund der Schäden) sowie eine Gefahrenanalyse (zukünftig zu erwartende Schäden) aufnehmen. Die überwiegende Mehrheit der Modelle und Präparate wurde nach Augenschein begutachtet. Für die wertvollen anatomischen Arm- und Beinstudien mussten, zur sicheren Klärung des Zustandes Röntgenaufnahmen ${ }^{14}$ angefertigt und die Materialzusammensetzung ${ }^{15}$ analytisch bestimmt werden. Für eine abschließende Gesamteinschätzung wurden sechs Zustandskategorien erstellt („stabil, ohne Schäden“ / „stabil, mit partiellen Schäden“ / „stabil, mit starken Schäden“ / „instabil, mit partiellen Schäden“ / „instabil, mit starken Schäden“ / „instabil, Notsicherung“). Die jeweils vergebene Schadenskategorie wurde in die Archivdatenbank eingetragen. Die Protokolle sowie allseitige Abbildungen ${ }^{16}$ mit Maßstab von jedem Objekt sind als PDF-Datei bzw. als JPG-Dateien ebenfalls über die Datenbank abrufbar.

Figur 4

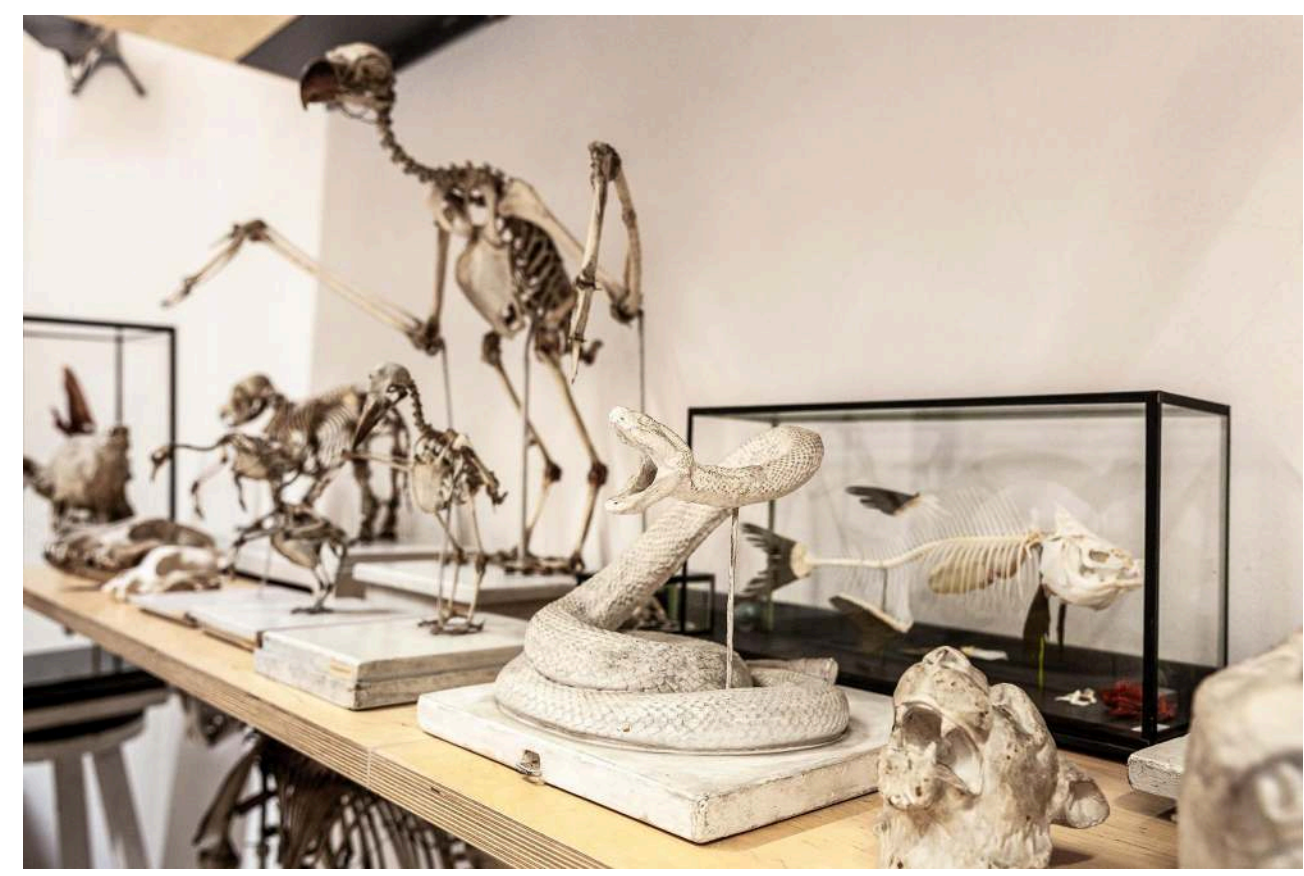

Modelle und Präparate zur Tieranatomie; im Vordergrund das Gipsmodell einer Schlange, hergestellt von der Kunstformerei Jos. Unger in Dresden.

(c) Robert Vanis.

Um die stabilen Objekte der Sammlung für den anatomischen Zeichenunterricht zur Verfügung stellen zu können und gleichzeitig alle instabilen Objekte zu schützen, wurden alle „instabil“ bewerteten Präparate und Modelle mit roten Metallplaketten gekennzeichnet - ein Sperrvermerk in situ. Als weitere Schutzmaßnahme schloss sich eine umfangreiche Überarbeitung der historischen Sockel an, deren Rollen größtenteils defekt oder schwergängig waren. Die historischen Rollen wurden demontiert und die originalen Sockel auf Hilfssockel mit leichtgängigen Kugellagerrollen positioniert [fig. 4]. Eine eigens entwickelte Zugvorrichtung sowie Drehteller [fig. 5] für kleine und mittelgroße Objekte ermöglichen eine berührungsfreie Bewegung im Raum und auf den hierfür konzipierten Zeichentischen. 


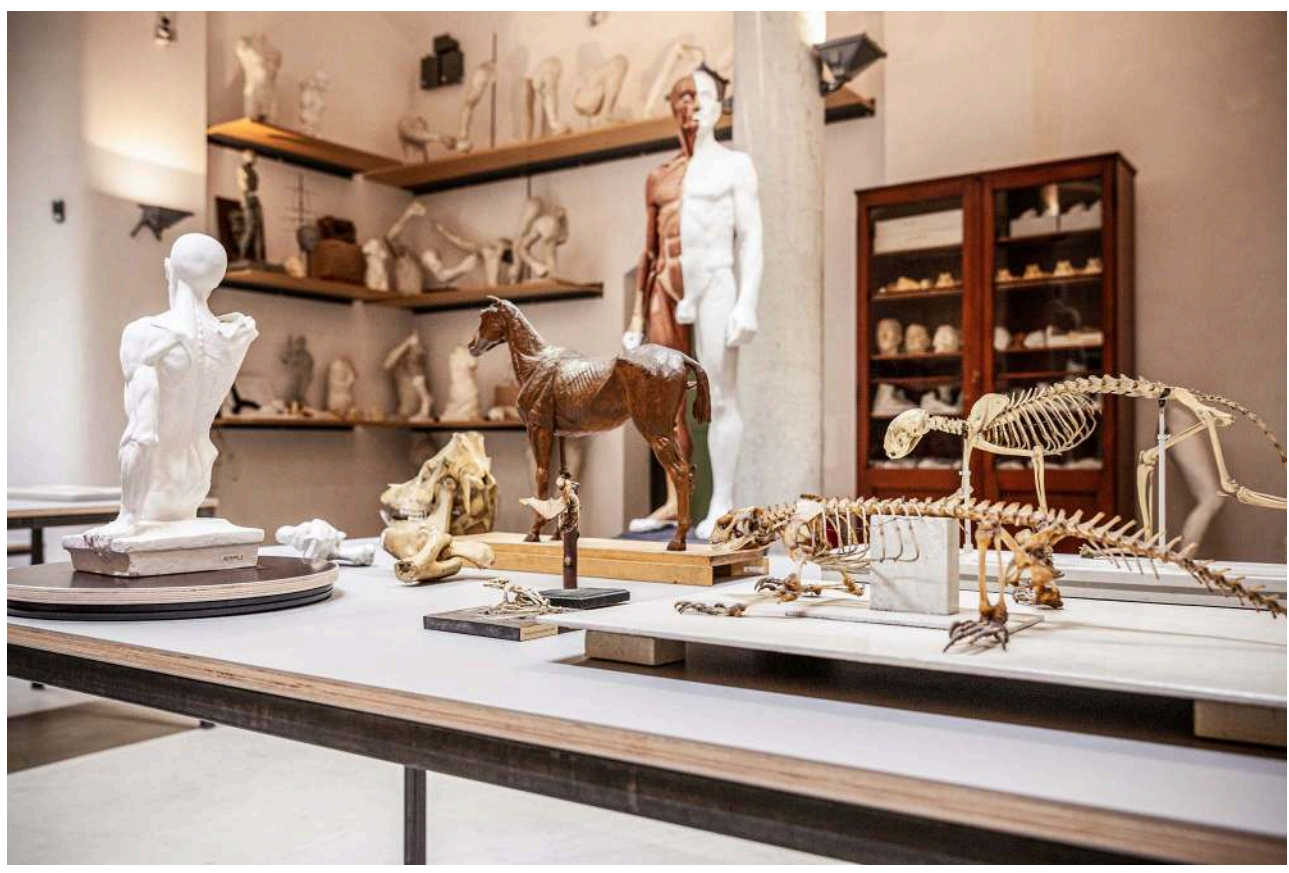

Modelle und Präparate zur Tieranatomie sowie ein humananatomisches Écorché in Gips; in der Bildmitte ein verkleinert dargestelltes anatomisches Pferdemodell in Plastilin, hergestellt, von Prof. Bammes zwischen 1975 und 1985.

(c) Robert Vanis.

Im Jahr 2018/19 erfolgte in Zusammenarbeit mit dem Studiengang „Kunsttechnologie, Konservierung und Restaurierung von Kunst und Kulturgut" der Hochschule die exemplarische Restaurierung eines der historischen Bänderskelette. Der Dornauszieher zeigte, wie alle Bänderskelette der Sammlung, signifikante Nutzungs- und Alterungsspuren und zudem Schäden, die bei einer Umsockelung entstanden waren. Neben konservatorischen und materialbezogenen Aspekten wurde der gesamte Restaurierungsprozess auch stets unter dem Gesichtspunkt eines ethisch korrekten Umgangs mit diesem Präparat betrachtet, womit die HfBK Dresden einen wichtigen Beitrag zur Erhaltung weiterer historischer menschlicher Präparate geleistet hat.

Die historischen Bänderskelette der HfBK Dresden wurden erstmals in den Jahren 2011 bis 2013 konservatorisch im Rahmen einer Masterarbeit in Kooperation mit der Königlich Dänischen Kunstakademie Kopenhagen (KADK) untersucht. Im Rahmen dieser Arbeit wurden an den Bänderskeletten zahlreiche Materialproben ${ }^{17}$ entnommen und Röntgenaufnahmen ${ }^{18}$ angefertigt. Anhand der Ergebnisse konnten die eingebrachten Materialien, wie trocknende öle und nachträglich eingesetzte Metallverbindungen (Reparaturmaßnahme), lokalisiert und identifiziert werden. Zudem zeigten die Laboranalysen, dass Fett aus dem Knocheninneren zum Teil massiv austritt und sich an der Oberfläche sammelt. Eine Veröffentlichung dieser Ergebnisse erfolgte 2018. ${ }^{19}$

Im Verlauf der aktuellen Restaurierungsmaßnahmen wurden zunächst zwei weitere Materialproben, davon eine an einem Metallstift (Korrosionsprodukt) aus dem Knie des Dornausziehers und eine vergleichend an einem Metalldraht (Korrosionsprodukt) eines stark nachfettenden Tierknochens entnommen. Die Analyse und Auswertung der Proben erfolgte im Labor für Archäometrie der HfBK Dresden..$^{20}$ Zur Anwendung kamen 
FT-IR-Sektrometrie5 (Fourier-Transform Infrarot-Spektrometer Fa. Bruker TENSOR 27 mit IR-Mikroskop HYPERION 2000, Diamantzelle bzw. ATR-Kristall Germanium) sowie Rasterelektronenmikroskopie und energiedispersiver Röntgenanalyse (REM/EDX) (REM Philips XL 30 mit Bruker EDX Detektor Xflash6130). Die Zusammensetzung der Korrosionsprodukte deutet darauf hin, dass die Fettsäuren aus dem Knocheninneren mit den Metalllegierungen reagiert haben, was zur Bildung grün-blauer Korrosionsprodukte (u.a. Kupfercarboxylat) geführt hat. Das sich durch Alterungsprozesse bildende saure Milieu auf der Knochenoberfläche und im Knocheninneren schädigt zum einen die natürlichen Kalziumverbindungen, aus denen die Knochen bestehen, zum anderen führen die Korrosionsprodukte zu irreversiblen Verfärbungen der Knochensubstanz. Aus diesen Gründen wurden die eingesetzten unedlen Metallverbindungen entfernt und durch Edelstahlmontagen sowie Verleimungen ersetzt.

Die Reinigung der Knochen, Kapseln und Bänder erfolgte unter Zuhilfenahme eines polaren Lösemittelgemischs aus Aceton und Ethanol, da eine wässrige Reinigung aufgrund der starken Degradation des organischen Gewebes ausgeschlossen werden musste. Ein Anquellen und Schrumpfen der Gewebefasern unter der Einwirkung von Wasser hätte irreversible Schäden und eine weitere Destabilisierung der Gelenkverbindungen verursachen können.

Aus ethischer und restaurierungsethischer Sicht wurde der Umgang mit fehlenden Körperteilen (fünf Zähne sowie zehn Finger- und Fußglieder) intensiv diskutiert. Auf Grundlage vorangegangener Überlegungen ${ }^{21}$ fiel die Entscheidung, alle verlorengegangenen Knochen und Zähne des Dornausziehers zu rekonstruieren und das Präparat somit anatomisch korrekt und dem ursprünglichen Erscheinungsbild entsprechend, präsentieren zu können. Die Rekonstruktion erfolgte mittels einer Mischung aus Wachs, Harz und Kreide; für die farbliche Integration wurden ölfarben verwendet. Eine weitere Rekonstruktionsmaßnahme betraf den Sockel des Präparates. Durch eine Überarbeitung war der Dornauszieher wesentlich zu niedrig "sitzend“ montiert worden, was eine stark verfälschte Körperhaltung zur Folge hatte. Ohne die historische Stützkonstruktion zu entfernen, wurde der Sockel leicht erhöht und eine Unterkonstruktion angefertigt, welche eine zukünftige Beschädigung überstehender Knochenteile verhindern soll. 
Figur 6

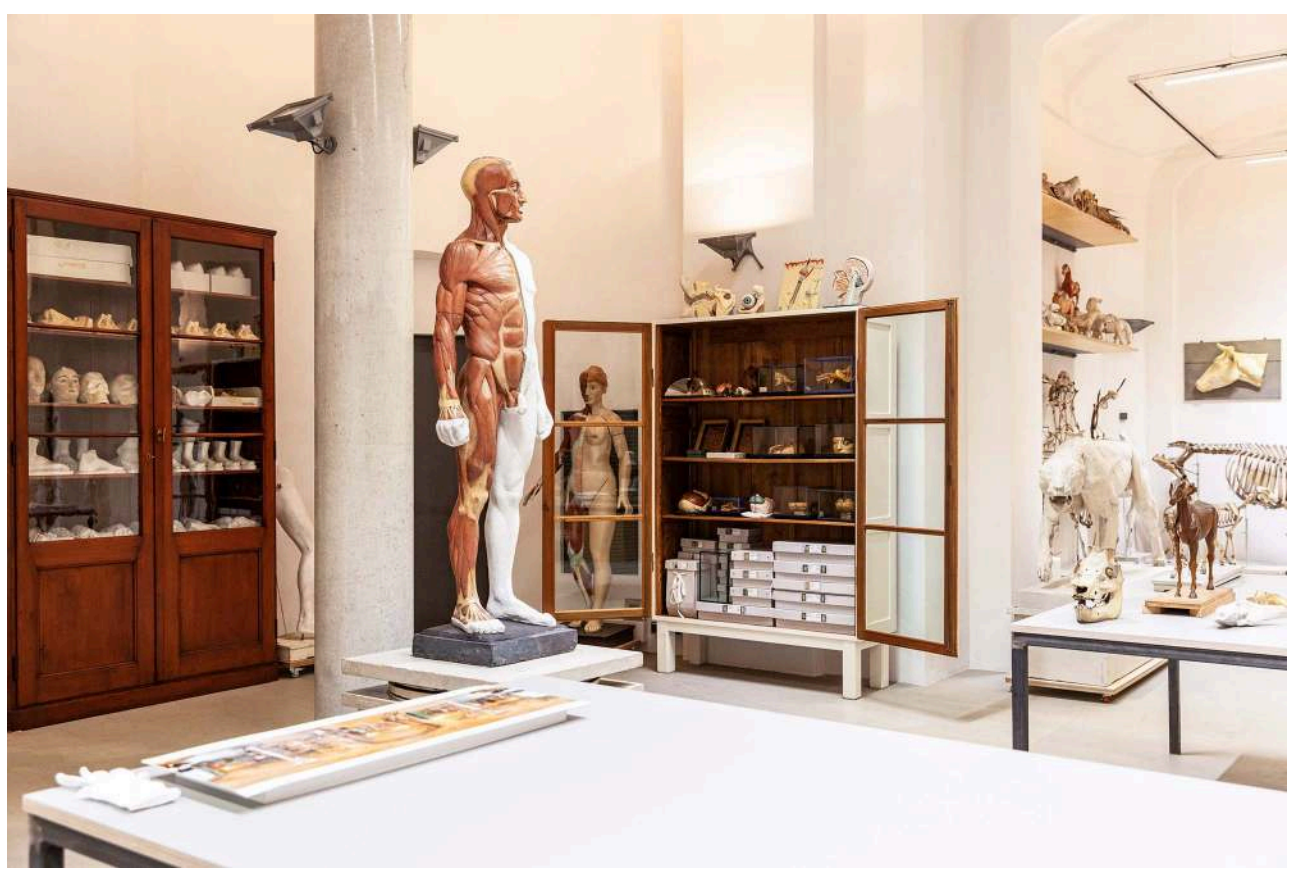

Blick in den Zeichensaal der Anatomischen Sammlung; zentral ein lebensgroßes farbig gefasstes Gipsmodell eines Muskelmannes, hergestellt von Franz Andreas Naumann, zwischen 1930 und 1935 (c) Robert Vanis.

Ebenfalls 2018 erfolgte eine zweite exemplarische Restaurierung im Rahmen des Projektes. Ein lebensgroßes Pferderelief aus Gips [fig. 6], welches nach derzeitigem Kenntnisstand in Kooperation mit der Königlich Sächsischen Thierärztlichen Hochschule entstand, bevor diese 1923 nach Leipzig umzog ${ }^{22}$, zeigte starke Beschädigungen und unsachgemäß ausgeführte Reparaturen, die das äußere Erscheinungsbild maßgeblich beeinträchtigten. Die Restaurierungsarbeiten wurden von externen Restauratoren ausgeführt. Neben Reinigungs- und Stabilisierungsmaßnahmen mussten die früheren Reparaturmaßnahmen bearbeitet werden. Konkret handelte es sich hierbei um Gips-Kittungen und sehr ungenau ausgeführte Retuschen, die die Anatomie des Modells in hohem Maße verfälschten. Durch gezielte Überarbeitungen der Kittungen und detailliert ausgeführte Retuschen konnten die optisch störenden Bereiche wieder in das Gesamterscheinungsbild des Modells integriert werden.

31 Einher gingen alle genannten Arbeitsschritte mit der Begutachtung der Lehrkonzepte des zeitgenössischen Anatomieunterrichts an der HfBK Dresden, denen die Lehrsammlung künftig verstärkt dienen wird. Darüber hinaus wurden aus der wissenschaftlichen Begutachtung interdisziplinäre Lehr- und Forschungsinhalte formuliert, vor allen für die Bereiche Kunst- und Sammlungsgeschichte sowie Konservierung / Restaurierung.

\section{Gemäldesammlung}

32 Die Gemäldesammlung in der Kustodie der HfBK Dresden ist zeitgeschichtliches Dokument und Kunstsammlung zugleich. Sie ist über die Jahrhunderte aus der Lehrtätigkeit der Institution entstanden und umfasst knapp 1500 Gemälde. 
Überwiegend stammen diese aus der Zeit der DDR, darunter viele Studien- und Diplomarbeiten, aber auch Schenkungen von emeritierten Professoren. Vereinzelt befinden sich auch ältere Werke in der Sammlung, von denen insbesondere die sogenannten Rezeptionsbilder hervorzuheben sind, mit denen sich Künstler an der Akademie um eine Lehrstelle bewarben. Entsprechend ist diese Gemäldesammlung nicht nur ein Zeugnis künstlerischer Lehrinhalte und -ergebnisse Dresdener Kunsthochschulgeschichte, sondern ebenso Zeugnis der wechselnden formalen und inhaltlichen Orientierungen in akademischer Lehre und künstlerischer Praxis in der DDR - von der Staatsgründung an bis zu ihrem Ende.

Über 350 Studierende erwarben in dieser Zeit einen Abschluss im Bereich Malerei, darunter so bekannte Künstlerinnen und Künstler wie Karl-Heinz Adler, Eberhard Göschel, Angela Hampel, Siegfried Klotz, Gerda Lepke, Harald Metzkes, Stefan Plenkers, Cornelia Schleime, Strawalde, Christoph Wetzel und Walter Womacka. Sie erhielten ihre Ausbildung unter anderem bei Professoren wie Rudolf Bergander, Gerhard Bondzin, Jutta Damme, Fritz Eisel, Hans Grundig, Wilhelm Lachnit, Heinz Lohmar und Paul Michaelis, die zum Teil selbst an der HfBK ausgebildet worden waren.

34 Für die kunstwissenschaftliche Auseinandersetzung mit der Malerei der DDR ist das Wissen um die Künstlerinnen und Künstler, ihre Ausbildung und Materialverwendung unter verschiedenen Fragestellungen unerlässlich. Da von vielen Dresdner Malereiabsolventen zwischen 1950 und 1990 mindestens eine Belegarbeit des Diploms vorliegt und somit die Abschlussergebnisse der Ausbildung des jeweiligen Künstlers fixiert sind, drängt sich die bis dato weitgehend unbekannte Sammlung als Forschungsgegenstand zur Erhellung der Kunstgeschichte der DDR geradezu auf. ${ }^{23}$

Zu Beginn des Projektes war die Aufbewahrung der Gemälde überwiegend prekär und eine Zugänglichkeit der Sammlung für Forschung und Öffentlichkeit nur eingeschränkt gegeben. Innerhalb des Projektes konnte dieser Zustand u.a. im Rahmen einer umfassenden Sommerschule ${ }^{24}$ im Jahr 2017 durch eine fachgerechte Bestands- und Zustandserfassung inklusive einer fotografischen Dokumentation (jeweils Vorder- und Rückseite mit Maßstab) sowie durch konservatorische und restauratorische Maßnahmen enorm verbessert werden. Darüber hinaus wurde ein Übersichtsplan des Gemäldedepots angefertigt sowie ein Standortverzeichnis angelegt.

36 Adäquat zur Anatomischen Sammlung wurde im Rahmen der sechswöchigen Sommerschule zunächst eine der oben genannten sechs Schadenskategorien für jedes erfasste Gemälde ermittelt und in der Archivdatenbank fixiert. Alle 263 Diplomgemälde sowie weitere ausgewählte Arbeiten wurden vertiefend untersucht, d.h. es erfolgte neben der Zustandseinschätzung auch eine konkrete Schadenserfassung und Ursachenanalyse. Teilweise wurden in diesem Arbeitsschritt auch Empfehlungen für spätere Restaurierungsmaßnahmen formuliert. Aufgrund der großen Anzahl zu untersuchender Gemälde konnte nicht für jedes Objekt ein separates Zustandsprotokoll angefertigt werden. Durch eine Modifizierung der Datenmaske war es dennoch möglich, alle gesammelten Informationen in der Archivdatenbank zu hinterlegen. ${ }^{25}$

Zusätzlich bzw. parallel zur Zustandserfassung fand die Durchführung präventiver Konservierungsmaßnahmen statt. In einem ersten Arbeitsschritt wurden die Gemäldegefache von Staub und Schmutz befreit und die Böden mit archivbeständigem Karton ausgelegt. Anschließend wurden die Gemälde nach Größe und Bildformat sortiert und wieder in die Gefache gestellt, wobei die Gemäldevorderseiten jeweils mit einer überstehenden Zwischenlage aus Archivkarton versehen wurden, um 
Beschädigungen der Malschichten auszuschließen. Während der Neubestückung der Gefache wurde darauf geachtet, dass die Inventarnummern auch ohne Bewegung der Gemälde einzusehen sind. Diejenigen Gemälde, welche in die Zustandskategorie „instabil, Notsicherung“ eingestuft wurden, erhielten eine besondere Markierung in der Archivdatenbank. Diese Arbeiten sollen zukünftig bevorzugt dem Studiengang Konservierung / Restaurierung der HfBK Dresden zur Verfügung gestellt werden und für Lehrveranstaltungen, Diplom- und Seminararbeiten genutzt werden. ${ }^{26}$

Maßgeblich erfolgten eine Aufarbeitung der Sammlungs- und Kunstgeschichte und eine Etablierung der Sammlung als Lehr- und Forschungsgegenstand für die oben genannten Disziplinen. Vor allem im Hinblick auf die in der DDR angewandten Maltechniken kann von den Ergebnissen des Projektes über die Grenzen Dresdens hinaus profitiert werden: So können fundierte, auf naturwissenschaftlichen und strahlentechnischen Analysen basierende Aussagen über Maltechniken in der DDR getroffen werden, die vor dem Hintergrund einer ständigen Mangelwirtschaft und der Suche nach Alternativen neue Erkenntnisse für die Wirtschafts- und Kulturgeschichte erwarten lassen und einen Beitrag zur Erhaltung von DDR-Kunst leisten.

\section{Präsentationen der Projektergebnisse}

Die Ergebnisse der Forschungsarbeit aus der Gemäldesammlung der HfBK Dresden wurden im Mai 2019 in der Ausstellung „Vor der Kunst. Malerei in der Kunsthochschule Dresden von 1950 bis 1990“ der Öffentlichkeit präsentiert. Über das Projekt hinaus zeigte die Kunstakademie St. Petersburg ausgewählte Arbeiten der Sammlung in einer Sonderausstellung im November 2019.

Im Rahmen der internationalen Tagung „Sammlungen an Kunsthochschulen. Speichern und Vermitteln" erfolgte am 06. November 2019 die feierliche Wiedereröffnung der Anatomischen Sammlung der HfBK Dresden. Seither findet wöchentlich der anatomische Zeichenunterricht für die Studierenden in den konservatorisch und baulich hergerichteten Räumlichkeiten statt. Für die interessierte öffentlichkeit ist die Sammlung zukünftig viermal im Semester nach vorheriger Anmeldung zugänglich; für Fachpublikum und Gruppen können zusätzlich individuelle Termine und Führungen vereinbart werden.

41 Eingebettet wurde der Festakt zur Eröffnung der Anatomischen Sammlung in ein dreitägiges Programm. Erstmalig wurden Sammlungen an europäischen Kunsthochschulen in den Blick genommen, ausgehend von der Frage, welche Hintergründe und welchen Stellenwert sie in den einzelnen Hochschulen haben, inwieweit das Speichern von künstlerischen Lehr-und Forschungsergebnissen praktiziert wird und hiernach eine Rückwirkung auf die Lehre erfolgt. Ziel war eine Bestandsaufnahme, um die reichhaltigen Potenziale der Sammlungen für Lehre, Forschung und Öffentlichkeit auszuloten, um etwaige Tendenzen zur Musealisierung zu hinterfragen und ihre Erhaltung sowie Anreicherung zu diskutieren.

Die Veröffentlichung des Tagungsbandes erfolgt Mitte 2020 und ist über die Website des Projektes (www.artonomia.de) abrufbar. Ebenfalls über diese Website sind zudem Handreichungen zur „Bestands- und Zustanderfassung von Gemälden sowie konservatorische Erstmaßnahmen in universitären Sammlungen“ und die Publikation 
„Menschliche Überreste im Depot. Empfehlungen für Betreuung und Nutzung“ herunterzuladen.

\section{NOTES}

1. Vgl. https://www.hfbk-dresden.de/hochschule/portraet/geschichte/ (17.04.2020) und MÜHLENBEREND Sandra, Surrogate der Natur. Die historische Anatomiesammlung der Kunstakademie Dresden. Paderborn, Fink, 2007,S. 32ff.

2. Vgl. PERES Constanze \& SCHMIDT Diether (dir.), Erneuerung als Tradition. 100 Jahre Dresdner Kunst und Kunstakademie im (inter)nationalen Zusammenhang, Dresden, Verlag der Kunst, 1997.

3. Vgl. MÜHLENBEREND Sandra, Surrogate der Natur.

4. Vgl. MÜHLENBEREND Sandra, Surrogate der Natur, S. $32 \mathrm{ff}$.

5. Vgl. MÜHLENBEREND Sandra, Surrogate der Natur, S. 44f.

6. Vgl. MÜHLENBEREND Sandra, «Präparate unter Verdacht. Künstleranatomie zwischen 1933-1945 an der Hochschule für Bildende Künste Dresden », in MÜHLENBEREND Sandra, FUCHS Jakob \& MARUŠIĆ Vera (dir.), Unmittelbarer Umgang mit menschlichen Überresten in Museen und Universitätssammlungen. Statements und Fallbeispiele, Dresde, HfBK, 2018, S.55ff, https:// wissenschaftliche-sammlungen.de/files/1815/4469/5645/Unmittelbarer-Umgang-mitmenschlichen-berresten-in-Museen-und-Universittssammlungen.pdf.

7. Bänderskelette wurden ursprünglich weitestgehend ohne die Nutzung künstlicher Hilfsmittel, wie Metalldrähten etc. hergestellt. Die natürlichen Gelenkverbindungen (Kapseln und Bänder) wurden hierfür am Knochen belassen.

8. Vgl. MÜHLENBEREND Sandra, Surrogate der Natur, S. $178 \mathrm{ff}$.

9. Vgl. MÜHLENBEREND Sandra, Surrogate der Natur, S. $154 \mathrm{ff}$.

10. Vgl. MÜHLENBEREND Sandra, Surrogate der Natur, S. $80 \mathrm{ff}$.

11. Vgl. MÜHLENBEREND Sandra, Surrogate der Natur, S. $222 \mathrm{ff}$.

12. Vgl. MÜHLENBEREND Sandra, Surrogate der Natur, S. $100 \mathrm{ff}$.

13. Vgl. MÜHLENBEREND Sandra, Surrogate der Natur.

14. Röntgenaufnahmen: Prof. Ivo Mohrmann und Kerstin Riße. (HfBK Dresden).

15. Analyse und Auswertung: Prof. Dr. Christoph Herm und Annegret Fuhrmann. (HfBK Dresden).

16. Abbildungen: Maria Katharina Franz. (HfBK Dresden).

17. Messungen und Auswertung: Prof. Dr. Christoph Herm, Annegret Fuhrmann, Dr. Sylvia Hoblyn. (HfBK Dresden).

18. Röntgenaufnahmen: Prof. Ivo Mohrmann, Kerstin Riße, Monika Kammer. (HfBK Dresden).

19. Vgl. FRANK Steen K., MEYER Ion, HERM Christoph \& BOTFELDT Knud B., "Status report, conservation and exhibition proposal for 14 natural skeletons from the anatomical collection at the University of Fine Arts in Dresden ", VDR Beiträge zur Erhaltung von Kunst- und Kulturgut, n 1 , 2018.

20. Messungen und Auswertung: Dr. Janine Kaden (HfBK Dresden).

21. Vgl. FUCHS Jakob, «Umgang mit fehlenden Körperteilen an historischen Skelettaufstellungen ", in MÜHLENBEREND Sandra, FUCHS Jakob \& MARUŠIĆ Vera (dir.), Unmittelbarer Umgang mit menschlichen Überresten...

22. Vgl. MÜHLENBEREND Sandra, Surrogate der Natur, S. $243 \mathrm{ff}$. 
23. Vgl. https://artonomia.de/wp-content/uploads/2019/05/Liste-derAbsolvent_innen-1947-1990_final.pdf (17.04.2020)

24. Leitung und Betreuung: Prof. Ivo Mohrmann und Kathleen Rosenthal. (HfBK Dresden).

25. Innerhalb des Projektes konnten somit alle Informationen, Untersuchungsergebnisse und Abbildungen des Gesamtbestandes „Anatomische Sammlung“ und „Gemäldesammlung“ in der AUGIAS-Archivdatenbank zusammengeführt werden.

26. Siehe auch: Arbeitsgruppe BMBF-Projekt „Körper und Malerei“ der Hochschule für Bildende Künste Dresden 2018.

\section{RÉSUMÉS}

Die Hochschule für Bildende Künste in Dresden (HfBK) ist neben der École nationale des beauxarts in Paris eine von zwei Kunstakademien in Europa, die über eine historische und sehr umfangreiche Sammlung zur Künstleranatomie verfügt. Die Anatomische Sammlung der HfBK Dresden wurde seit Beginn des 19. Jahrhunderts kontinuierlich zusammengetragen und erweitert. Sie umfasst heute ca. 700 anatomische Modelle und Präparate zur Human- und Tieranatomie. Mit Hilfe der zahlreichen historischen Präparate und Modelle entwickelten die Professoren für den anatomischen Zeichenunterricht im 20. Jahrhundert eigenen didaktische Modelle und Lehrmethoden, die in ihrer Bedeutung weit über die Grenzen der HfBK Dresden hinausgingen. Nach 1989 verloren die historischen Modelle und Präparate stark an Bedeutung; die Sammlung wurde kaum mehr für den Unterricht genutzt und ihr Erhaltungszustand verschlechterte sich. Erst Ende der 1990er Jahre wurde der enorme Wert der Sammlung wiedererkannt und erste Maßnahmen zu ihrem Schutz und ihrer Erforschung eingeleitet. Zunächst wurde die Sammlung vollständig aus dem aktiven Lehrbetrieb herausgenommen und in einen Depotraum verbracht. Seither steht ihr Bestand im Fokus der kunsthistorischen und konservatorischen Erforschung. Von Februar 2017 bis Januar 2020 fand ein vom Bundesministerium für Bildung und Forschung gefördertes Projekt statt, mit dem Ziel, die Anatomische Sammlung nach vielen Jahren wieder für die Lehre zur Verfügung zu stellen. Am 06.11.2019 fand nach dreijähriger intensiver Forschungs- und Konzeptarbeit die feierliche Wiedereröffnung statt.

Dresden University of Fine Arts (HfBK) is, with the École nationale des beaux-arts de Paris, one of the two academies of Fine Arts in Europe that possesses an old and very rich collection for artistic anatomy. The anatomy collection of Dresden University of Fine Arts has been built up since the beginning of the 20th century and constantly expanded. It comprises today nearly 700 anatomic models and specimens of human and animal anatomy. Thanks to those numerous specimens and ancient models, teachers developed in the 20th century their own educational material and methods whose value had been recognized far beyond the frontiers of the HfBK Dresden. After 1989, the old models and specimens lost their importance; the collection was hardly used for teaching and its condition had deteriorated. It was only in the late 1990's that the immense value of the collection was rediscovered, and that the first protective measures were taken and the first studies were carried out. The collection was first removed completely from active teaching material and placed in a storage room. Since then, those fonds have been the focus of research in art history and conservation. From February 2017 to January 2020, a project financed by the Federal Ministry of Education and Research aimed at making the collection 
available again for teaching, after all those years. On 6 November 2019, after three years of intensives research and projects, its reopening was celebrated.

\section{INDEX}

Schlüsselwörter : Hochschule für Bildende Künste Dresden, Körper und Malerei, anatomische Modelle, Anatomische Sammlung, Zeichnung, Lehre, Dresden, Malerei, Gemäldesammlung Keywords : University of Fine Arts of Dresden, Körper und Malerei, anatomic models, anatomical preparation, drawing, teaching, Dresden, painting, painting collection

\section{AUTEURS}

\section{SANDRA MÜHLENBEREND}

Chef de projet et coordinatrice « Körper und Malerei » (février 2017-janvier 2020), École des beaux-arts de Dresde sandra.muehlenberend@web.de

\section{JAKOB FUCHS}

Assistant scientifique, projet « Körper und Malerei » (février 2017-janvier 2020), École des beauxarts de Dresde

fuchs@hfbk-dresden.de

\section{IVO MOHRMANN}

Président du comité de pilotage de projet « Körper und Malerei » (février 2017-janvier 2020), chef du département de Technologie de l'art, d'Examen radiologique et de Photographie, conservateur des collections universitaires, École des Beaux-Arts de Dresde mohrmann@hfbk-dresden.de 